\title{
The Arabidopsis cytosolic proteome: the metabolic heart of the cell
}

\author{
Jun Ito ${ }^{1,2}$, Harriet T. Parsons ${ }^{1,2,3}$ and Joshua L. Heazlewood ${ }^{1,2} *$ \\ 1 Joint BioEnergy Institute, Emeryville, CA, USA \\ 2 Physical Biosciences Division, Lawrence Berkeley National Laboratory, Berkeley, CA, USA \\ ${ }^{3}$ Department of Plant and Environmental Sciences, University of Copenhagen, Copenhagen, Denmark
}

\section{Edited by:}

Nicolas L. Taylor, The University of Western Australia, Australia

\section{Reviewed by:}

Ján A. Miernyk, University of Missouri, USA

Stefanie Wienkoop, University of Vienna, Austria

\section{*Correspondence:}

Joshua L. Heazlewood, Joint BioEnergy Institute, Lawrence Berkeley National Laboratory, One Cyclotron Road MS 978-4466, Berkeley, CA 94720, USA e-mail: jlheazlewood@lbl.gov
The plant cytosol is the major intracellular fluid that acts as the medium for inter-organellar crosstalk and where a plethora of important biological reactions take place. These include its involvement in protein synthesis and degradation, stress response signaling, carbon metabolism, biosynthesis of secondary metabolites, and accumulation of enzymes for defense and detoxification. This central role is highlighted by estimates indicating that the majority of eukaryotic proteins are cytosolic. Arabidopsis thaliana has been the subject of numerous proteomic studies on its different subcellular compartments. However, a detailed study of enriched cytosolic fractions from Arabidopsis cell culture has been performed only recently, with over 1,000 proteins reproducibly identified by mass spectrometry. The number of proteins allocated to the cytosol nearly doubles to 1,802 if a series of targeted proteomic characterizations of complexes is included. Despite this, few groups are currently applying advanced proteomic approaches to this important metabolic space. This review will highlight the current state of the Arabidopsis cytosolic proteome since its initial characterization a few years ago.

Keywords: cytosol, ribosome, proteasome, localization, Arabidopsis

\section{INTRODUCTION}

The cytosol is the liquid portion of a cell that contains principle cellular constituents comprising membrane-bound organelles. The cytosol itself lacks membrane compartmentalization. Within its highly concentrated aqueous setting of dissolved ionic solutes, small molecule metabolites and macromolecules, which include nucleic acids and proteins, a wide range of biochemical reactions are known to occur. These include an involvement in glycolysis (Plaxton, 1996), the oxidative branch of the pentose phosphate pathway (Schnarrenberger etal., 1995), protein biosynthesis and degradation (Bailey-Serres et al., 2009; Vierstra, 2009), signal transduction (Lecourieux et al., 2006; Klimecka and Muszynska, 2007), primary and secondary metabolite biosynthesis and transportation (Lundmark et al., 2006; Lunn, 2007; Martinoia et al., 2007; Weber and Fischer, 2007; Krueger et al., 2009), stress response signaling (Yamada and Nishimura, 2008; Cazale etal., 2009; Sugio et al., 2009), and the accumulation of enzymes for defense and detoxification (Laule etal., 2003; Dixon etal., 2009; Sappl et al., 2009). Furthermore, nuclearencoded organellar proteins are synthesized in the cytosol prior to their import into organelles by targeting peptides (Jarvis, 2008; Prassinos etal., 2008; Huang etal., 2009). Although the cytosol has a multitude of prominent biochemical processes in the eukaryotic cell (Figure 1), only two proteome surveys have been carried to date on the plant cytosol. The first study identified 69 abundant proteins in cytosolic samples of soybean root nodules (Oehrle et al., 2008) while the second study identified 1,071 proteins from a large-scale mass spectrometry (MS) analysis of cytosol-enriched fractions from Arabidopsis thaliana cell suspension cultures (Ito et al., 2010). Many of the identified proteins were from well-known cytosolic processes (Figure 1); although a significant portion of the functionally unclassifiable proteins likely undertake novel roles in the cytosol (Ito et al., 2010). In this review, we will discuss further developments that have occurred from these initial proteomic analyses of the Arabidopsis cytosol.

\section{THE Arabidopsis CYTOSOLIC 80S RIBOSOME}

The cytosolic ribosome is a major component of the Arabidopsis cytosol and has been targeted by a number of studies for analysis by proteomics. A significant proportion of the proteins identified in the cytosolic proteome of Arabidopsis are involved in the core biological process of protein biosynthesis and degradation (Book etal., 2010; Ito et al., 2010; Hummel et al., 2012). The ribosome was well-represented amongst these proteins, with 92 previously identified ribosomal protein subunits from 61 of the 80 gene families (Ito et al., 2010). Arabidopsis ribosomal proteins have highly conserved sequences that belong to small gene families of two to six members, most of which are expressed (Carroll et al., 2008). A total of 79 of the 80 ribosomal protein families were characterized in purified ribosome preparations from Arabidopsis leaves (Giavalisco et al., 2005) and cell suspension cultures (Chang et al., 2005; Carroll et al., 2008). This included the identification of post-translational modifications (PTMs) such as initiator methionine removal, N-terminal acetylation, $\mathrm{N}$-terminal methylation, lysine $\mathrm{N}$-methylation, and phosphorylation. These studies represent basic proteomic surveys of the ribosome; more recent analyses have undertaken quantitative approaches to characterize this important protein complex of the cytosol. 


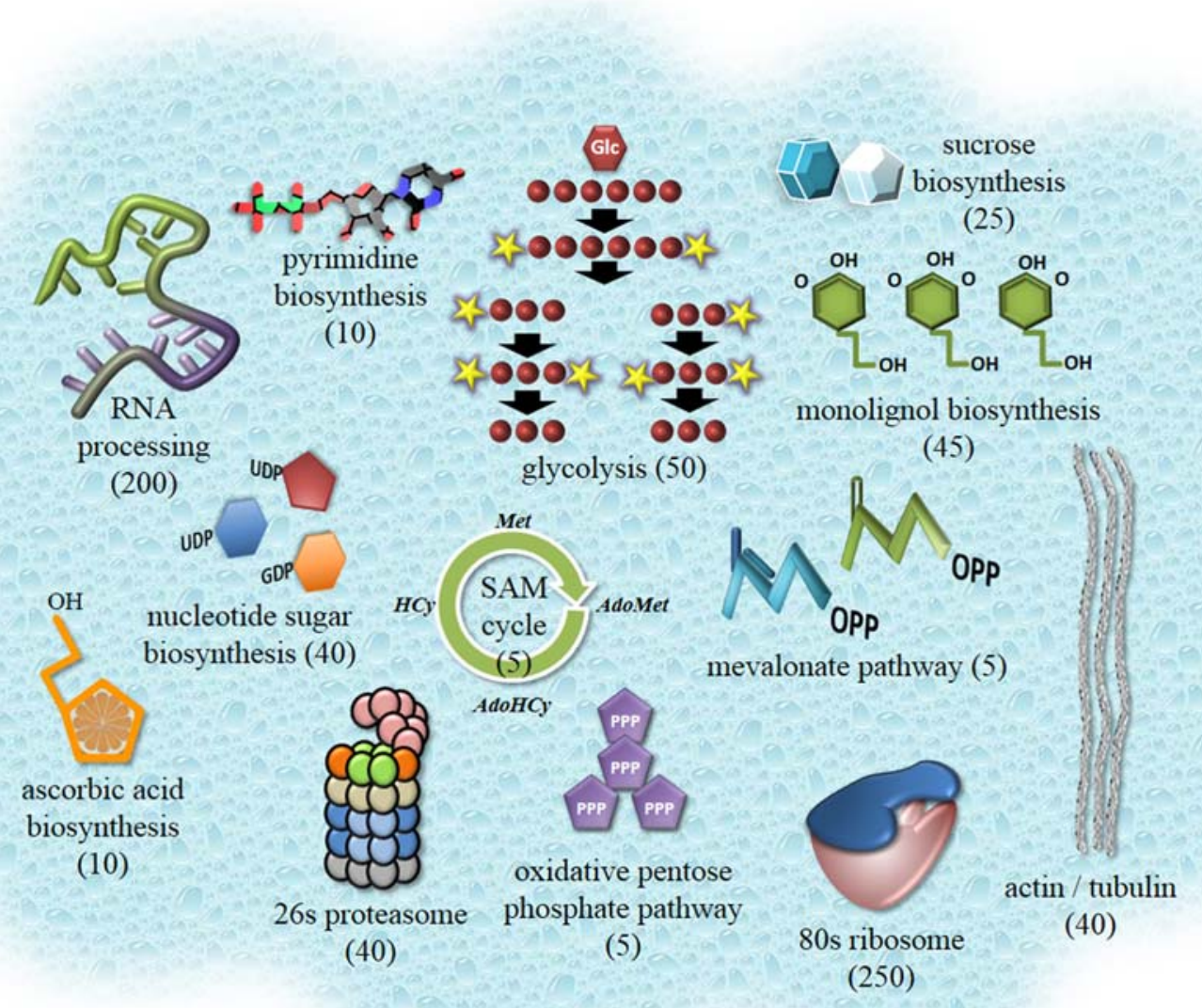

FIGURE 1 | Cartoon highlighting prominent metabolic processes, proteins, and protein complexes of the plant cytosol. Components were selected based on the proteomic data outlined in supplementary material of Ito etal. (2010). The number of proteins contributing to each of these components is shown in brackets. This information was obtained from Plant Metabolic Network resource (Zhang et al., 2005) and from MapMan pathway dataset for Arabidopsis (Thimm et al., 2004). Collectively, over 2,600 proteins can be assigned to the Arabidopsis cytosol when considering proteomics studies, localization with fluorescent proteins and functional curation.
Two quantitative proteomic studies have attempted to measure changes in the Arabidopsis ribosomal proteome under defined growing conditions. The first quantitative study investigated differential phosphorylation of purified ribosomal proteins from Arabidopsis leaves at day and night cycles as a possible mechanism to regulate diurnal protein synthesis (Turkina et al., 2011). Phosphorylation was detected by liquid chromatography (LC)MS/MS on eight serine residues of six ribosomal proteins: S2-3, S6-1, S6-2, P0-2, P1, and L29-1. Relative quantification of phosphopeptides by differential stable isotope labeling and LC-MS/MS showed significant increases in day to night phosphorylation ratios of ribosomal proteins S6 at Ser-231 (2.2-fold), S6-1 and S6-2 variants at Ser-240 (4.2- and 1.8-fold, respectively), and L29-1 at Ser-58 (1.6-fold). This indicated that differential phosphorylation of these ribosomal proteins are likely mechanisms in modulating diurnal translation in plants (Turkina et al., 2011). The second study performed a label-free absolute quantitative analysis by $\mathrm{LC}-\mathrm{MS}^{\mathrm{E}}$ of immune-purified ribosomal protein paralogs from transgenic Arabidopsis leaves in response to sucrose feeding - a treatment known to have a profound effect on plant physiology and gene regulation (Hummel et al., 2012). The extensive families of ribosomal protein paralogs, the ambiguity of their incorporation into ribosomes and the potential alterations to ribosome composition in response to environmental and developmental cues were all factors in carrying out this study. Indeed, out of 204 ribosomal proteins identified by LC-MS/MS, 13 paralogs including S8A, S3aA, L12C, L19A-C, L30B, L8C, L28A, S12A, S12C, L22B, and S7C, as well as the ribosomal scaffold protein RACK1A, showed significant changes in their abundances up to 2.7-fold by LC-MS ${ }^{\mathrm{E}}$ in response to sucrose treatments (Hummel et al., 2012). While L28A, L19A, and RACK1 have been shown to be important in normal plant growth and development (Tzafrir et al., 2004; Chen etal., 2006; Yao etal., 2008), the majority display limited phenotypic traits in their mutant plants. Concurrently, multiple ribosomal protein paralogs were shown to be incorporated into ribosomes in both sucrose fed and unfed 
plants. It was surmised from these results that the Arabidopsis cytosolic ribosomes undergo variable alteration to their protein paralog compositions in reaction to changing external conditions (Hummel et al., 2012).

\section{THE Arabidopsis CYTOSOLIC 26S PROTEASOME}

The $26 \mathrm{~S}$ proteasome is a complex of approximately $2.5 \mathrm{MDa}$ which is responsible for the proteolytic degradation of most ubiquitylated proteins. Ubiquitylated protein degradation regulates processes such as the cell cycle, organ morphogenesis, circadian rhythms, and environmental response (Vierstra, 2009). The proteasome consists of a 28 -subunit core protease $(\mathrm{CP})$, which houses the active sites for protein and peptide hydrolysis, and a regulatory particle (RP) of at least 18 subunits which regulates substrate recognition, unfolding, and access to the $\mathrm{CP}$. The architecture is highly conserved amongst eukaryotes but recent affinity purification of the 26S complex from Arabidopsis has revealed that although the plant $26 \mathrm{~S}$ proteasome is analogous to that of the human and yeast (Kim et al., 2011), important differences exist.

In Arabidopsis, as in other plant groups, almost all subunits in both the CP and RP are encoded by duplicate genes of at least $90 \%$ homology, of which few appear to be pseudogenes (Book et al., 2010). Complexes containing all subunit duplicants have been purified from whole plants and characterized by MS (Yang et al., 2004; Book et al., 2010). It is not known yet whether duplicants are inserted into the $26 \mathrm{~S}$ proteasome randomly or specifically. If these subunit "duplicants" are functionally specific, this raises the possibility of localized regulation of specific protein groups by populations of $26 \mathrm{~S}$ proteasomes containing specific subunit duplicants/variants. In mutant backgrounds for the RPT2a/b subunit (Lee et al., 2011), complementation studies revealed functional redundancy between duplicants. However, double rpt2a/rpt2b knockout mutants exhibited a more severe phenotype that either single mutant, suggesting redundancy is only partial. RPN2a has uniquely been shown to be unregulated in response to increased sucrose concentrations, implicating a RPN2a-complex in the degradation hexokinase signaling pathway proteins (Sun et al., 2012). Likewise, single RPN5a/b mutants are phenotypically different and double mutants are lethal (Book et al., 2009; Serino and Pick, 2013). Together, these pieces of evidence point toward neofunctionalization of gene duplicants, supporting the idea of multiple populations of complexes within a whole plant.

Most of what is known about the plant 26S proteasome comes from yeast studies and has been reviewed previously (Finley, 2009; Vierstra, 2009). However, a recent study of RPN10 in Arabidopsis shows that important functional differences exist, at least in recognition of ubiquitylated substrates (Lin et al., 2011). Further unique properties of the Arabidopsis $26 \mathrm{~S}$ proteasome include a much greater degree of ubiquitylation of subunits than has been observed in yeast (Peng et al., 2003; Book etal., 2010). Subunits became ubiquitylated when still assembled as a complex, implying that this modification performed a function beyond tagging subunits for degradation after complex disassembly. Accessory proteins help assemble the complex and recognize and recruit ubiquitylated substrates. A number of proteins homologous to yeast accessory proteins co-purified with the Arabidopsis $26 \mathrm{~S}$ proteasome, as well as some novel putative accessory proteins not found in yeast (Book etal., 2010). An interesting question for future studies is whether certain accessory proteins associate with particular subunit variants/ duplicants.

An important aim in understanding plant $26 \mathrm{~S}$ proteasome function is to understand the relationship between subunit composition, and specific protein degradation in response to changes in internal and external environments. Given the high identity of many of these subunits, this will involve a significant challenge for characterization by MS. Nonetheless, together with the recent analysis of the ubiquitylated proteome in Arabidopsis (Kim et al., 2013), such work will undoubtedly expand our understanding of signaling and process regulation related to this important cytosolic protein complex.

\section{POST-TRANSLATIONAL MODIFICATIONS}

The ability to routinely identify and quantify PTMs represents a grand challenge in the field of proteomics (Heazlewood, 2011). However, few proteomic studies have targeted a subcellular compartment to specifically characterize PTMs (de la Fuente van Bentem etal., 2006; Ito et al., 2009). To the best of our knowledge, no such survey has ever been conducted on highly purified cytosolic fractions from Arabidopsis. Aside from the detailed analyses of the purified cytosolic complexes $80 \mathrm{~S}$ ribosome and $26 \mathrm{~S}$ proteasome outlined above, PTMs identified on cytosolic localized proteins are largely the result of large-scale PTM-targeted studies. In Arabidopsis, this has included phosphorylation (Heazlewood et al., 2008), $N$-linked glycosylation (Zielinska et al., 2012), ubiquitination (Kim et al., 2013), methionine oxidation (Marondedze et al., 2013), S-nitrosylation (Fares et al., 2011), and acetylation (Finkemeier et al., 2011). With few exceptions, these studies comprise collections of identified sites and do not generally explore the functional implication of a PTM. However, a number of more detailed investigations have identified the importance of PTMs on proteins localized to the cytosol. Entry into the cytosolic oxidative pentose phosphate pathway (OPPP) is catalyzed by glucose-6-phosphate dehydrogenase (G6PD) which is encoded by AT3G27300 and AT5G40760 in Arabidopsis. Large-scale phosphoproteomic studies have identified phosphorylation sites on both cytosolic isoforms. Recently it was demonstrated that the phosphorylation of AT5G40760 at Thr-467 increased G6PD activity fourfold (Dal Santo et al., 2012). Glycolysis represents a key metabolic pathway in the plant cytosol. The sixth step in this pathway is catalyzed by glyceraldehyde-3-phosphate dehydrogenase (GAPDH) and represents the beginning of a net gain in ATP and NADH. In Arabidopsis, the step is encoded by a small gene family, a member of which has been identified as lysine acetylated (AT1G13440) in Arabidopsis. It was also demonstrated that the acetylation of Lys-130 inhibited the activity of this enzyme in vitro and consequently this PTM may represent a regulatory mechanism for this step in the pathway (Finkemeier et al., 2011). GAPDH encoded by AT1G13440 also contains $N$-glycosylation and numerous phosphorylation sites according to a number of targeted PTM studies (Heazlewood et al., 2008; Zielinska et al., 2012). The functional roles, if any, of the many thousands of 
PTMs on cytosolic localized proteins will likely take many years to accurately characterize. Recently many of these sites were incorporated into the MASCP Gator, the Arabidopsis proteomics aggregation portal (Mann etal., 2013). It is envisaged that the inclusion of this information into such a utility will enable the community to better leverage these data for future functional analyses.

\section{UTILIZATION OF THE Arabidopsis CYTOSOLIC PROTEOME}

Establishing the subcellular location of a protein is an important factor in determining its function (Chou and Cai, 2003). MS analysis of purified organelles or cellular compartments and chimeric fluorescent fusion proteins are two common experimental methods used to define subcellular localizations of Arabidopsis proteins (Heazlewood et al., 2007; Tanz et al., 2013). Over 2,200 proteins contain information indicating a cytosolic localization in Arabidopsis (Table 1), which comprises nearly 25\% of all experimentally localized proteins in the SUBcellular Arabidopsis database (SUBA). A large proportion of these cytosolic proteins have been identified in multiple subcellular compartments, especially in the case of proteomic approaches. It is therefore ideal, though often not the case, that protein localization is confirmed using complementary methods (Millar et al., 2009).

Several recent reports have used data from the Arabidopsis cytosolic proteome to confirm functional interpretations supporting a localization in the cytosol. Overall, they exemplify the practicality of this subcellular proteome for verifying the cytosolic localizations of different proteins. Glyoxylate reductase (GLYR) is a central enzyme in the $\gamma$-aminobutyrate (GABA) metabolic pathway, where it catalyzes the detoxification of glyoxylate and succinic semialdehdye (Ching et al., 2012). The two plant isoforms GLYR1 and GLYR2 were believed to localize to the cytosol or peroxisomes, and plastid, respectively. Conflicting reports of Arabidopsis GLYR1 (At3g25530) localizing in the cytosol (Simpson et al., 2008) or the peroxisome (Reumann et al., 2009) had implications for defining its exact metabolic roles and the compartmentation of the GABA and photorespiratory pathways. This was resolved by visualizing $\mathrm{N}$-terminal green fluorescent protein (GFP)-tagged GLYR1 in Arabidopsis suspension-cultured cells, leaves and seedlings and tobacco BY-2 suspension-cultured cells, where it was observed to exclusively localize in the cytosol (Ching et al., 2012). Its identification by MS as a major

Table 1 | A survey of cytosolic proteins experimentally localized in Arabidopsis from the SUBA database as of November 2013 (Tanz et al., 2013).

\begin{tabular}{lllll}
\hline & MS/MS & FP & Total & Overlap \\
\hline All locations & 7891 & 2647 & 9319 & 1219 \\
Cytosol & 1808 & 580 & 2262 & 126
\end{tabular}

MS/MS indicates proteins identified through subcellular proteomics studies; FP are proteins localized using a fluorescent protein tag. The overlap between FP and MS/MS for cytosolic proteins is significantly worse than all proteins localized in the SUBA database. Possibly reflecting poor attention to this subcellular space and its processes by the research community. protein in the cytosolic proteome of Arabidopsis cell suspensions was cited as further evidence of this finding (Ito et al., 2010; Ching et al., 2012).

The Arabidopsis translation elongation factor eEF-1B $\beta 1$ (EF1B $\beta$, At1g30230) is involved in plant cell wall biosynthesis and it is essential for normal plant development (Hossain et al., 2012). Arabidopsis plants with T-DNA insertions in their EF1B $\beta$ gene display a dwarf phenotype, with alterations to their vascular morphology and inflorescence stem structures and 38 and 20\% reductions in total lignin and crystalline cellulose content, respectively. By transforming Arabidopsis plants with a $35 \mathrm{~S}$ promoter-controlled $\mathrm{EF} 1 \mathrm{~B} \beta$ fused with yellow fluorescent protein $(\mathrm{EF} 1 \mathrm{~B} \beta-\mathrm{YFP})$, the subcellular locations of EF1B $\beta$ were visualized in the plasma membrane and cytosol (Hossain et al., 2012). These observations agreed with MS analyses of the Arabidopsis plasma membrane (Mitra et al., 2009) and cytosol proteomes (Ito etal., 2010), with EF1B $\beta$ identified in both subcellular compartments.

An evolutionary and structural analysis of a human disrupted in schizophrenia 1 (DISC1) protein conducted orthology searches of non-vertebrate reference organisms such as Dictyostelium, Trichoplax, Monosiga, and Arabidopsis (Sanchez-Pulido and Ponting, 2011). This study found that while most DISC1 orthologs lacked any experimental evidence of their functions, the Arabidopsis DISC1 ortholog (At5g25070) is ubiquitously expressed in various tissues and developmental stages and is a constituent of the Arabidopsis cytosolic proteome (Ito et al., 2010). This was strikingly similar to human DISC1, which is expressed in a wide range of tissues and also cytosol-localized (Sanchez-Pulido and Ponting, 2011).

\section{EXPANDING THE Arabidopsis CYTOSOLIC PROTEOME}

A computational analysis of the Arabidopsis proteome estimated that the cytosolic proteome may contain around 5,400 \pm 650 proteins (Ito et al., 2010). This indicates that the current experimental set of 2,262 proteins likely represents only about $40 \%$ of the cytosolic proteome (Table 1). A dissection of fluorescent protein-based localization studies of Arabidopsis proteins (Table 1) reveals that many members were also identified in the Arabidopsis cytosolic proteome (recent examples include Ching et al., 2012; Christ et al., 2012; Hossain etal., 2012; Li et al., 2012; Lu et al., 2012; McLoughlin et al., 2012; Witz et al., 2012). However, there are many examples of FP-tagged proteins that have been localized to the cytosol and not identified by proteomic surveys (some recent studies include Gaber et al., 2012; Hernandez et al., 2012; Kwon et al., 2012; Lu et al., 2012; McLoughlin et al., 2012; Rautengarten et al., 2012; Vadassery et al., 2012; Witz et al., 2012). The inclusion of complementary subcellular datasets such as those available from the gene ontology database AmiGO (Carbon et al., 2009) and UniProtKB (Magrane and UniProt Consortium, 2011) can also be used to capture some of these missing cytosolic proteins. Nearly 2000 Arabidopsis proteins are designated as cytosolic by AmiGO, while about 1,300 Arabidopsis proteins are allocated to the cytosol by the UniProt Protein Knowledgebase. Incorporating these data with the proteomic and fluorescent protein information, the total number of Arabidopsis proteins with some cytosolic designation is 2604 distinct members or about $50 \%$ of the computationally 
derived proteome. It should be noted that the "experimental" figure of ca. 2,600 does not account for false positives resulting from proteins with multiple subcellular designations. Over 1,400 of these proteins also have non-cytosolic assignments by either MS or fluorescent protein localizations according to SUBA (Tanz et al., 2013).

While proteomics has identified a considerable proportion of the computationally derived cytosolic proteome (around 30\%), the shortfall can be readily explained and include: many proteins are not abundant and thus not easily detected by MS, many proteins could be expressed in tissue(s) other than cell suspension cultures or only under certain conditions (i.e., at a specific stage of plant development or in response to stress) and most significantly only one out of the nearly 120 proteomic analyses of various subcellular compartments from Arabidopsis has been performed on its cytosolic fraction (Heazlewood et al., 2007; Ito et al., 2010). In contrast, studies in Arabidopsis in the areas of respiration and photosynthesis have benefited tremendously from the characterization of their proteomes across different organs and tissues, developmental stages, and growth conditions (Lee et al., 2008; van Wijk and Baginsky, 2011). In order to better understand its dynamics, future analyses of the Arabidopsis cytosolic proteome will also need to reach this level of diversity.

A critical factor in performing in-depth proteomic analysis of the cytosol from plants will be to obtain relatively pure cytosolic fractions from this material. Isolating the cytosolic fraction from Arabidopsis cell suspensions relies on enzymatic generation of protoplasts and their disruption by gentle pressure to maintain organelle integrity, followed by organelle removal by differential centrifugation (Ito et al., 2010). Unlike uniform heterotrophic cell suspensions, cytosol purification from plants requires extra steps including the removal of chloroplasts. A study of protein localization between cytosol and chloroplasts of Arabidopsis seedlings developed a method for isolating the cytosolic fraction from protoplasts of seedlings (Estavillo et al., 2011). The addition of density centrifugation was necessary to remove broken protoplasts and intact chloroplasts, respectively, from the seedling cytosolic fraction (Estavillo et al., 2011, 2014). Employing immunoblotting or MS-based quantitation against subcellular markers to assess organelle contamination during the extraction process (Ito et al., 2010), this method could be further refined to generate highpurity cytosolic fractions from many types of Arabidopsis plant material for proteomic analysis.

Sub-fractionation of the cytosol is an effective way to reduce its protein complexity and to improve MS/MS identification of low abundant cytosolic proteins. Unlike mitochondria and plastids, the cytosol lacks defined membrane-bound compartments that can be further sub fractionated (Eubel et al., 2007; Ferro et al., 2010). However, isolating soluble protein complexes from the Arabidopsis cytosol has been shown to be relatively straight forward. As outlined above, both the $80 \mathrm{~S}$ ribosome and the $26 \mathrm{~S}$ proteasome have been isolated and extensively characterized by MS (Yang et al., 2004; Chang et al., 2005; Giavalisco et al., 2005; Carroll et al., 2008; Book et al., 2010; Turkina et al., 2011; Hummel et al., 2012). Beyond these examples, sub-fractionation of other cytosolic protein groups will likely rely on affinity purification techniques tailored to the physiochemical properties of target proteins to simplify complex mixtures and enrich for low abundant proteins. In non-plant systems approaches have included immobilized heparin chromatography to fractionate cytosolic proteins from human breast cancer MCF-7 cells (Shefcheck et al., 2003). Approximately 300 low-abundant cytosolic proteins were detected by two-dimensional gel electrophoresis (2-DE) of heparin fractions, and they were not present on 2-DE separations of total cytosolic protein mixtures (Shefcheck et al., 2003). Finally, LC-MS/MS analysis of tandem biomimetic affinity pre-fractionation of rat liver cytosol proteins identified 665 unique rat proteins, which was significantly more than the 371 proteins in the unfractionated cytosol (Tan et al., 2009).

\section{PERSPECTIVES}

There is tremendous scope to extend our current knowledge of the multitude of reactions that take place in the plant cytosol. Few studies have employed quantitative proteomic approaches to study cytosolic components revealing a lack of attention to this important compartment. Similarly, the characterization and analysis of PTMs of cytosolic proteins will be a significant challenge in the future. Recent reports of cytosolic localizations of Arabidopsis proteins by fluorescent protein tagging showed that while a number of them were identified in the cytosolic proteome, many others were not. Future comparative analysis of cytosolic proteomes of different plant tissues grown under various environmental conditions is essential to better understand its dynamics and to unravel its complexity. Isolating pure cytosolic fractions and their subfractions from diverse sources of plant material for LC-MS/MS analysis will be key factors to achieve this aim.

\section{AUTHOR CONTRIBUTIONS}

The manuscript was devised by Jun Ito and written by Jun Ito, Harriet T. Parsons, and Joshua L. Heazlewood. Figure and Table were constructed by Joshua L. Heazlewood.

\section{ACKNOWLEDGMENTS}

This work was part of the DOE Joint BioEnergy Institute (http://www.jbei.org) supported by the U.S. Department of Energy, Office of Science, Office of Biological and Environmental Research, through contract DE-AC02-05CH11231 between Lawrence Berkeley National Laboratory and the U.S. Department of Energy. Harriet T. Parsons was supported by a Marie Curie Fellowship.

\section{REFERENCES}

Bailey-Serres, J., Sorenson, R., and Juntawong, P. (2009). Getting the message across: cytoplasmic ribonucleoprotein complexes. Trends Plant Sci. 14, 443-453. doi: 10.1016/j.tplants.2009.05.004

Book, A. J., Gladman, N. P., Lee, S. S., Scalf, M., Smith, L. M., and Vierstra, R. D. (2010). Affinity purification of the Arabidopsis $26 \mathrm{~S}$ proteasome reveals a diverse array of plant proteolytic complexes. J. Biol. Chem. 285, 25554-25569. doi: 10.1074/jbc.M110.136622

Book, A. J., Smalle, J., Lee, K.-H., Yang, P., Walker, J. M., Casper, S., et al. (2009). The RPN5 subunit of the 26s proteasome is essential for gametogenesis, sporophyte development, and complex assembly in Arabidopsis. Plant Cell 21, 460-478. doi: 10.1105/tpc.108.064444

Carbon, S., Ireland, A., Mungall, C. J., Shu, S., Marshall, B., Lewis, S., et al. (2009). AmiGO: online access to ontology and annotation data. Bioinformatics 25, 288289. doi: 10.1093/bioinformatics/btn615 
Carroll, A. J., Heazlewood, J. L., Ito, J., and Millar, A. H. (2008). Analysis of the Arabidopsis cytosolic ribosome proteome provides detailed insights into its components and their post-translational modification. Mol. Cell. Proteomics 7, 347-369. doi: 10.1074/mcp.M700052-MCP200

Cazale, A. C., Clement, M., Chiarenza, S., Roncato, M. A., Pochon, N., Creff, A., et al. (2009). Altered expression of cytosolic/nuclear HSC70-1 molecular chaperone affects development and abiotic stress tolerance in Arabidopsis thaliana. J. Exp. Bot. 60, 2653-2664. doi: 10.1093/jxb/erp109

Chang, I. F., Szick-Miranda, K., Pan, S., and Bailey-Serres, J. (2005). Proteomic characterization of evolutionarily conserved and variable proteins of Arabidopsis cytosolic ribosomes. Plant Physiol. 137, 848-862. doi: 10.1104/pp.104. 053637

Chen, J. G., Ullah, H., Temple, B., Liang, J., Guo, J., Alonso, J. M., et al. (2006). RACK1 mediates multiple hormone responsiveness and developmental processes in Arabidopsis. J. Exp. Bot. 57, 2697-2708. doi: 10.1093/jxb/erl035

Ching, S. L., Gidda, S. K., Rochon, A., Van Cauwenberghe, O. R., Shelp, B. J., and Mullen, R. T. (2012). Glyoxylate reductase isoform 1 is localized in the cytosol and not peroxisomes in plant cells. J. Integr. Plant Biol. 54, 152-168. doi: 10.1111/j.1744-7909.2012.01103.x

Chou, K. C., and Cai, Y. D. (2003). Prediction and classification of protein subcellular location-sequence-order effect and pseudo amino acid composition. J. Cell. Biochem. 90, 1250-1260. doi: 10.1002/jcb.10719

Christ, B., Schelbert, S., Aubry, S., Sussenbacher, I., Muller, T., Krautler, B., et al. (2012). MES16, a member of the methylesterase protein family, specifically demethylates fluorescent chlorophyll catabolites during chlorophyll breakdown in Arabidopsis. Plant Physiol. 158, 628-641. doi: 10.1104/pp.111.188870

Dal Santo, S., Stampfl, H., Krasensky, J., Kempa, S., Gibon, Y., Petutschnig, E., et al (2012). Stress-induced GSK3 regulates the redox stress response by phosphorylating glucose-6-phosphate dehydrogenase in Arabidopsis. Plant Cell 24, 3380-3392. doi: $10.1105 /$ tpc.112.101279

de la Fuente van Bentem, S., Anrather, D., Roitinger, E., Djamei, A., Hufnagl, T. Barta, A., et al. (2006). Phosphoproteomics reveals extensive in vivo phosphorylation of Arabidopsis proteins involved in RNA metabolism. Nucleic Acids Res. 34, 3267-3278. doi: 10.1093/nar/gkl429

Dixon, D. P., Hawkins, T., Hussey, P. J., and Edwards, R. (2009). Enzyme activities and subcellular localization of members of the Arabidopsis glutathione transferase superfamily. J. Exp. Bot. 60, 1207-1218. doi: 10.1093/jxb/ern365

Estavillo, G. M., Crisp, P. A., Pornsiriwong, W., Wirtz, M., Collinge, D., Carrie, C., et al. (2011). Evidence for a SAL1-PAP chloroplast retrograde pathway that functions in drought and high light signaling in Arabidopsis. Plant Cell 23, 39924012. doi: 10.1105/tpc.111.091033

Estavillo, G. M., Verhertbruggen, Y., Scheller, H. V., Pogson, B. J., Heazlewood, J. L., and Ito, J. (2014). Isolation of the plant cytosolic fraction for proteomic analysis Methods Mol. Biol. 1072, 453-467. doi: 10.1007/978-1-62703-631-3_31

Eubel, H., Heazlewood, J. L., and Millar, A. H. (2007). "Isolation and subfractionation of plant mitochondria for proteomic analysis," in Plant Proteomics: Methods and Protocols, eds H. Thiellement, M. Zivy, C. Damerval, and V. Méchin. (Totowa, NJ: Humana Press Inc.), 49-62

Fares, A., Rossignol, M., and Peltier, J. B. (2011). Proteomics investigation of endogenous S-nitrosylation in Arabidopsis. Biochem. Biophys. Res. Commun. 416, 331-336. doi: 10.1016/j.bbrc.2011.11.036

Ferro, M., Brugière, S., Salvi, D., Seigneurin-Berny, D., Court, M., Moyet, L., et al. (2010).AT_CHLORO, a comprehensive chloroplast proteome database with subplastidial localization and curated information on envelope proteins. Mol. Cell. Proteomics 9, 1063-1084. doi: 10.1074/mcp.M900325-MCP200

Finkemeier, I., Laxa, M., Miguet, L., Howden, A. J. M., and Sweetlove, L. J. (2011) Proteins of diverse function and subcellular location are lysine acetylated in Arabidopsis. Plant Physiol. 155, 1779-1790. doi: 10.1104/pp.110.171595

Finley, D. (2009). Recognition and processing of ubiquitin-protein conjugates by the proteasome. Annu. Rev. Biochem. 78, 477-513. doi: 10.1146/annurev.biochem.78.081507.101607

Gaber, A., Ogata, T., Maruta, T., Yoshimura, K., Tamoi, M., and Shigeoka, S. (2012). The involvement of Arabidopsis glutathione peroxidase 8 in the suppression of oxidative damage in the nucleus and cytosol. Plant Cell Physiol. 53, 1596-1606 doi: $10.1093 / \mathrm{pcp} / \mathrm{pcs} 100$

Giavalisco, P., Wilson, D., Kreitler, T., Lehrach, H., Klose, J., Gobom, J., et al. (2005). High heterogeneity within the ribosomal proteins of the Arabidopsis thaliana $80 \mathrm{~S}$ ribosome. Plant Mol. Biol. 57, 577-591. doi: 10.1007/s11103-005-0699-3
Heazlewood, J. L. (2011). The green proteome: challenges in plant proteomics. Front. Plant Sci. 2:6. doi: 10.3389/fpls.2011.00006

Heazlewood, J. L., Durek, P., Hummel, J., Selbig, J., Weckwerth, W., Walther, D., et al. (2008). PhosPhAt: a database of phosphorylation sites in Arabidopsis thaliana and a plant-specific phosphorylation site predictor. Nucleic Acids Res. 36, D1015D1021

Heazlewood, J. L., Verboom, R. E., Tonti-Filippini, J., Small, I., and Millar, A. H. (2007). SUBA: the Arabidopsis Subcellular Database. Nucleic Acids Res. 35, D213-D218. doi: 10.1093/nar/gkl863

Hernandez, L., Whitehead, L., He, Z., Gazda, V., Gilday, A., Kozhevnikova, E., et al. (2012). A cytosolic acyltransferase contributes to triacylglycerol synthesis in sucrose-rescued Arabidopsis seed oil catabolism mutants. Plant Physiol. 160, 215-225. doi: 10.1104/pp.112.201541

Hossain, Z., Amyot, L., Mcgarvey, B., Gruber, M., Jung, J., and Hannoufa, A. (2012) The translation elongation factor eEF-1B $\beta 1$ is involved in cell wall biosynthesis and plant development in Arabidopsis thaliana. PLoS ONE 7:e30425. doi: 10.1371/journal.pone.0030425

Huang, S., Taylor, N. L., Whelan, J., and Millar, A. H. (2009). Refining the definition of plant mitochondrial presequences through analysis of sorting signals, N-terminal modifications, and cleavage motifs. Plant Physiol. 150, 1272-1285. doi: 10.1104/pp.109.137885

Hummel, M., Cordewener, J. H., De Groot, J. C., Smeekens, S., America, A. H., and Hanson, J. (2012). Dynamic protein composition of Arabidopsis thaliana cytosolic ribosomes in response to sucrose feeding as revealed by label free MSE proteomics. Proteomics 12, 1024-1038. doi: 10.1002/pmic.201100413

Ito, J., Batth, T. S., Petzold, C. J., Redding-Johanson, A. M., Mukhopadhyay, A., Verboom, R., et al. (2010). Analysis of the Arabidopsis cytosolic proteome highlights subcellular partitioning of central plant metabolism. J. Proteome Res. 10, 1571-1582. doi: 10.1021/pr1009433

Ito, J., Taylor, N. L., Castleden, I., Weckwerth, W., Millar, A. H., and Heazlewood, J. L. (2009). A survey of the Arabidopsis thaliana mitochondrial phosphoproteome. Proteomics 9, 4229-4240. doi: 10.1002/pmic.200900064

Jarvis, P. (2008). Targeting of nucleus-encoded proteins to chloroplasts in plants. New Phytol. 179, 257-285. doi: 10.1111/j.1469-8137.2008.02452.x

Kim, D. Y., Scalf, M., Smith, L. M., and Vierstra, R. D. (2013). Advanced proteomic analyses yield a deep catalog of ubiquitylation targets in Arabidopsis. Plant Cell 25, 1523-1540. doi: 10.1105/tpc.112.108613

Kim, H. M., Yu, Y., and Cheng, Y. (2011). Structure characterization of the 26S proteasome. Biochim. Biophys. Acta 1809, 67-79. doi: 10.1016/j.bbagrm.2010.08.008

Klimecka, M., and Muszynska, G. (2007). Structure and functions of plant calciumdependent protein kinases. Acta Biochim. Pol. 54, 219-233

Krueger, S., Niehl, A., Lopez Martin, M. C., Steinhauser, D., Donath, A., Hildebrandt, T., et al. (2009). Analysis of cytosolic and plastidic serine acetyltransferase mutants and subcellular metabolite distributions suggests interplay of the cellular compartments for cysteine biosynthesis in Arabidopsis. Plant Cell Environ. 32, 349-367. doi: 10.1111/j.1365-3040.2008.01928.x

Kwon, Y., Yu, S. I., Lee, H., Yim, J. H., Zhu, J. K., and Lee, B. H. (2012). Arabidopsis serine decarboxylase mutants implicate the roles of ethanolamine in plant growth and development. Int. J. Mol. Sci. 13, 3176-3188. doi: 10.3390/ijms130 33176

Laule, O., Furholz, A., Chang, H. S., Zhu, T., Wang, X., Heifetz, P. B., et al. (2003). Crosstalk between cytosolic and plastidial pathways of isoprenoid biosynthesis in Arabidopsis thaliana. Proc. Natl. Acad. Sci. U.S.A. 100, 6866-6871. doi: $10.1073 /$ pnas. 1031755100

Lecourieux, D., Ranjeva, R., and Pugin, A. (2006). Calcium in plant defence-signalling pathways. New Phytol. 171, 249-269. doi: 10.1111/j.14698137.2006.01777.x

Lee, C. P., Eubel, H., O’Toole, N., and Millar, A. H. (2008). Heterogeneity of the mitochondrial proteome for photosynthetic and non-photosynthetic Arabidopsis metabolism. Mol. Cell. Proteomics 7, 1297-1316. doi: 10.1074/mcp.M700535MCP200

Lee, K. H., Minami, A., Marshall, R. S., Book, A. J., Farmer, L. M., Walker, J. M., et al. (2011). The RPT2 subunit of the $26 \mathrm{~S}$ proteasome directs complex assembly, histone dynamics, and gametophyte and sporophyte development in Arabidopsis. Plant Cell 23, 4298-4317. doi: 10.1105/tpc.111.089482

Li, H. M., Chen, H., Yang, Z. N., and Gong, J. M. (2012). Cdi gene is required for pollen germination and tube growth in Arabidopsis. FEBS Lett. 586, 1027-1031. doi: 10.1016/j.febslet.2012.02.046 
Lin, Y. L., Sung, S. C., Tsai, H. L., Yu, T. T., Radjacommare, R., Usharani, R., et al. (2011). The defective proteasome but not substrate recognition function is responsible for the null phenotypes of the Arabidopsis proteasome subunit RPN10. Plant Cell 23, 2754-2773. doi: 10.1105/tpc.111.086702

Lu, W., Tang, X., Huo, Y., Xu, R., Qi, S., Huang, J., et al. (2012). Identification and characterization of fructose 1,6-bisphosphate aldolase genes in Arabidopsis reveal a gene family with diverse responses to abiotic stresses. Gene 503, 65-74. doi: 10.1016/j.gene.2012.04.042

Lundmark, M., Cavaco, A. M., Trevanion, S., and Hurry, V. (2006). Carbon partitioning and export in transgenic Arabidopsis thaliana with altered capacity for sucrose synthesis grown at low temperature: a role for metabolite transporters. Plant Cell Environ. 29, 1703-1714. doi: 10.1111/j.1365-3040.2006.01543.x

Lunn, J. E. (2007). Compartmentation in plant metabolism. J. Exp. Bot. 58, 35-47. doi: $10.1093 / \mathrm{jxb} / \mathrm{erl} 134$

Magrane, M., and UniProt Consortium. (2011). UniProt Knowledgebase: a hub of integrated protein data. Database 2011, bar009. doi: 10.1093/database/bar009

Mann, G. W., Calley, P. C., Joshi, H. J., and Heazlewood, J. L. (2013). MASCP gator: an overview of the Arabidopsis proteomic aggregation portal. Front. Plant Sci 4:411. doi: 10.3389/fpls.2013.00411

Marondedze, C., Turek, I., Parrott, B., Thomas, L., Jankovic, B., Lilley, K. S., et al. (2013). Structural and functional characteristics of cGMP-dependent methionine oxidation in Arabidopsis thaliana proteins. Cell Commun. Signal. 11, 1. doi: 10.1186/1478-811X-11-1

Martinoia, E., Maeshima, M., and Neuhaus, H. E. (2007). Vacuolar transporters and their essential role in plant metabolism. J. Exp. Bot. 58, 83-102. doi: $10.1093 / \mathrm{jxb} / \mathrm{erl} 183$

McLoughlin, F., Galvan-Ampudia, C. S., Julkowska, M. M., Caarls, L., Van Der Does, D., Lauriere, C., et al. (2012). The Snf1-related protein kinases SnRK2.4 and SnRK2.10 are involved in maintenance of root system architecture during salt stress. Plant J. 72, 436-449. doi: 10.1111/j.1365-313X.2012.05089.x

Millar, A. H., Carrie, C., Pogson, B., and Whelan, J. (2009). Exploring the functionlocation nexus: using multiple lines of evidence in defining the subcellular location of plant proteins. Plant Cell 21, 1625-1631. doi: 10.1105/tpc.109.066019

Mitra, S. K., Walters, B. T., Clouse, S. D., and Goshe, M. B. (2009). An efficient organic solvent based extraction method for the proteomic analysis of Arabidopsi plasma membranes. J. Proteome Res. 8, 2752-2767. doi: 10.1021/pr801044y

Oehrle, N. W., Sarma, A. D., Waters, J. K., and Emerich, D. W. (2008). Proteomic analysis of soybean nodule cytosol. Phytochemistry 69, 2426-2438. doi: 10.1016/j.phytochem.2008.07.004

Peng, J., Schwartz, D., Elias, J. E., Thoreen, C. C., Cheng, D., Marsischky, G., et al. (2003). A proteomics approach to understanding protein ubiquitination. Nat. Biotechnol. 21, 921-926. doi: 10.1038/nbt849

Plaxton, W. C. (1996). The organization and regulation of plant glycolysis. Annu. Rev. Plant Physiol. Plant Mol. Biol. 47, 185-214. doi 10.1146/annurev.arplant.47.1.185

Prassinos, C., Haralampidis, K., Milioni, D., Samakovli, D., Krambis, K., and Hatzopoulos, P. (2008). Complexity of Hsp90 in organelle targeting. Plant Mol. Biol. 67, 323-334. doi: 10.1007/s11103-008-9322-8

Rautengarten, C., Ebert, B., Ouellet, M., Nafisi, M., Baidoo, E. E., Benke, P., et al. (2012). Arabidopsis deficient in cutin ferulate encodes a transferase required for feruloylation of omega-hydroxy fatty acids in cutin polyester. Plant Physiol. 158, 654-665. doi: 10.1104/pp.111.187187

Reumann, S., Quan, S., Aung, K., Yang, P., Manandhar-Shrestha, K., Holbrook, D., et al. (2009). In-depth proteome analysis of Arabidopsis leaf peroxisomes combined with in vivo subcellular targeting verification indicates novel metabolic and regulatory functions of peroxisomes. Plant Physiol. 150, 125-143. doi: 10.1104/pp.109.137703

Sanchez-Pulido, L., and Ponting, C. P. (2011). Structure and evolutionary history of DISC1. Hum. Mol. Genet. 20, R175-R181. doi: 10.1093/hmg/ddr374

Sappl, P. G., Carroll, A. J., Clifton, R., Lister, R., Whelan, J., Millar, A. H., et al. (2009). The Arabidopsis glutathione transferase gene family displays complex stress regulation and co-silencing multiple genes results in altered metabolic sensitivity to oxidative stress. Plant J. 58, 53-68. doi: 10.1111/j.1365-313X.2008. 03761.x

Schnarrenberger, C., Flechner, A., and Martin, W. (1995). Enzymatic evidence for a complete oxidative pentose phosphate pathway in chloroplasts and an incomplete pathway in the cytosol of spinach leaves. Plant Physiol. 108, 609-614. doi: 10.1104/pp.108.2.609
Serino, G., and Pick, E. (2013). Duplication and familial promiscuity within the proteasome lid and COP9 signalosome kin complexes. Plant Sci. 203, 89-97. doi: 10.1016/j.plantsci.2012.12.018

Shefcheck, K., Yao, X., and Fenselau, C. (2003). Fractionation of cytosolic proteins on an immobilized heparin column. Anal. Chem. 75, 1691-1698. doi: $10.1021 / \mathrm{ac} 026153 \mathrm{~h}$

Simpson, J. P., Di Leo, R., Dhanoa, P. K., Allan, W. L., Makhmoudova, A., Clark, S. M., et al. (2008). Identification and characterization of a plastid-localized Arabidopsis glyoxylate reductase isoform: comparison with a cytosolic isoform and implications for cellular redox homeostasis and aldehyde detoxification. J. Exp. Bot. 59, 2545-2554. doi: 10.1093/jxb/ern 123

Sugio, A., Dreos, R., Aparicio, F., and Maule, A. J. (2009). The cytosolic protein response as a subcomponent of the wider heat shock response in Arabidopsis. Plant Cell 21, 642-654. doi: 10.1105/tpc.108.062596

Sun, H. H., Sako, K., Suzuki, Y., Maekawa, S., Yasuda, S., Chiba, Y., et al. (2012). Sugar-inducible RPT2a, a subunit of 26S proteasome, participates in sugar response in Arabidopsis. Plant Biotechnol. 29, 279-284. doi: 10.5511/plantbiotechnology.12.0409a

Tan, Q., Dong, D., Ye, L., Huo, C., Huang, F., and Li, R. (2009). Pre-fractionation of rat liver cytosol proteins prior to mass spectrometry-based proteomic analysis using tandem biomimetic affinity chromatography. J. Mol. Recognit. 23, 93-100. doi: 10.1002/jmr.995

Tanz, S. K., Castleden, I., Hooper, C. M., Vacher, M., Small, I., and Millar, H. A. (2013). SUBA3: a database for integrating experimentation and prediction to define the SUBcellular location of proteins in Arabidopsis. Nucleic Acids Res. 41, D1185-D1191. doi: 10.1093/nar/gks1151

Thimm, O., Blasing, O., Gibon, Y., Nagel, A., Meyer, S., Kruger, P., et al. (2004). MAPMAN: a user-driven tool to display genomics data sets onto diagrams of metabolic pathways and other biological processes. Plant J. 37, 914-939. doi: 10.1111/j.1365-313X.2004.02016.x

Turkina, M. V., Klang Arstrand, H., and Vener, A. V. (2011). Differential phosphorylation of ribosomal proteins in Arabidopsis thaliana plants during day and night. PLoS ONE 6:e29307. doi: 10.1371/journal.pone.0029307

Tzafrir, I., Pena-Muralla, R., Dickerman, A., Berg, M., Rogers, R., Hutchens, S., et al. (2004). Identification of genes required for embryo development in Arabidopsis. Plant Physiol. 135, 1206-1220. doi: 10.1104/pp.104.045179

Vadassery, J., Reichelt, M., Hause, B., Gershenzon, J., Boland, W., and Mithofer, A (2012). CML42-mediated calcium signaling coordinates responses to Spodoptera herbivory and abiotic stresses in Arabidopsis. Plant Physiol. 159, 1159-1175. doi: 10.1104/pp.112.198150

van Wijk, K. J., and Baginsky, S. (2011). Plastid proteomics in higher plants: current state and future goals. Plant Physiol. 155, 1578-1588. doi: 10.1104/pp.111. 172932

Vierstra, R. D. (2009). The ubiquitin-26S proteasome system at the nexus of plant biology. Nat. Rev. Mol. Cell Biol. 10, 385-397. doi: 10.1038/nrm2688

Weber, A. P., and Fischer, K. (2007). Making the connections - the crucial role of metabolite transporters at the interface between chloroplast and cytosol. FEBS Lett. 581, 2215-2222. doi: 10.1016/j.febslet.2007.02.010

Witz, S., Jung, B., Furst, S., and Mohlmann, T. (2012). De novo pyrimidine nucleotide synthesis mainly occurs outside of plastids, but a previously undiscovered nucleobase importer provides substrates for the essential salvage pathway in Arabidopsis. Plant Cell 24, 1549-1559. doi: 10.1105/tpc.112.096743

Yamada, K., and Nishimura, M. (2008). Cytosolic heat shock protein 90 regulates heat shock transcription factor in Arabidopsis thaliana. Plant Signal. Behav. 3, 660-662. doi: 10.4161/psb.3.9.5775

Yang, P., Fu, H., Walker, J., Papa, C. M., Smalle, J., Ju, Y. M., et al. (2004). Purification of the Arabidopsis $26 \mathrm{~S}$ proteasome: biochemical and molecular analyses revealed the presence of multiple isoforms. J. Biol. Chem. 279, 6401-6413. doi: 10.1074/jbc.M311977200

Yao, Y., Ling, Q., Wang, H., and Huang, H. (2008). Ribosomal proteins promote leaf adaxial identity. Development 135, 1325-1334. doi: 10.1242/dev.017913

Zhang, P., Foerster, H., Tissier, C. P., Mueller, L., Paley, S., Karp, P. D., et al. (2005). MetaCyc and AraCyc. Metabolic pathway databases for plant research. Plant Physiol. 138, 27-37. doi: 10.1104/pp.105.060376

Zielinska, D. F., Gnad, F., Schropp, K., Wisniewski, J. R., and Mann, M. (2012). Mapping $N$-glycosylation sites across seven evolutionarily distant species reveals a divergent substrate proteome despite a common core machinery. Mol. Cell 46, 542-548. doi: 10.1016/j.molcel.2012.04.031 
Conflict of Interest Statement: The authors declare that the research was conducted in the absence of any commercial or financial relationships that could be construed as a potential conflict of interest.

Received: 16 November 2013; accepted: 19 January 2014; published online: 05 February 2014.

Citation: Ito J, Parsons HT and Heazlewood JL (2014) The Arabidopsis cytosolic proteome: the metabolic heart of the cell. Front. Plant Sci. 5:21. doi: 10.3389/fpls. 2014.00021
This article was submitted to Plant Proteomics, a section of the journal Frontiers in Plant Science.

Copyright (c) 2014 Ito, Parsons and Heazlewood. This is an open-access article distributed under the terms of the Creative Commons Attribution License (CCBY). The use, distribution or reproduction in other forums is permitted, provided the original author(s) or licensor are credited and that the original publication in this journal is cited, in accordance with accepted academic practice. No use, distribution or reproduction is permitted which does not comply with these terms. 